\title{
Sleep patterns and sugar-sweetened beverage consumption among children from around the world
}

\author{
Jean-Philippe Chaput ${ }^{1,2, *}$, Mark S Tremblay ${ }^{1,2}$, Peter T Katzmarzyk ${ }^{3}$, Mikael Fogelholm ${ }^{4}$, \\ Gang Hu${ }^{3}$, Carol Maher ${ }^{5}$, Jose Maia ${ }^{6}$, Timothy Olds ${ }^{5}$, Vincent Onywera ${ }^{7}$, Olga L Sarmiento ${ }^{8}$, \\ Martyn Standage 9 , Catrine Tudor-Locke ${ }^{3,10}$ and Hugues Sampasa-Kanyinga ${ }^{2}$ for the \\ ISCOLE Research Group \\ 'Healthy Active Living and Obesity Research Group, Children's Hospital of Eastern Ontario Research Institute, 401 \\ Smyth Road, Ottawa, Canada, K1H 8L 1: ${ }^{2}$ Faculty of Medicine, University of Ottawa, Ottawa, Canada: ${ }^{3}$ Pennington \\ Biomedical Research Center, Baton Rouge, LA, USA: ${ }^{4}$ Department of Food and Environmental Sciences, University of \\ Helsinki, Helsinki, Finland: ${ }^{5}$ School of Health Sciences, University of South Australia, Adelaide, Australia: ${ }^{6} \mathrm{CIFI} 2 \mathrm{D}$, \\ Faculdade de Desporto, University of Porto, Porto, Portugal: ${ }^{7}$ Department of Recreation Management and Exercise \\ Science, Kenyatta University, Nairobi, Kenya: ${ }^{8}$ School of Medicine, Universidad de los Andes, Bogota, Colombia: \\ ${ }^{9}$ Department of Health, University of Bath, Bath, UK: ${ }^{10}$ Department of Kinesiology, University of Massachusetts \\ Amherst, Amherst, MA, USA
}

Submitted 30 June 2017: Final revision received 18 December 2017: Accepted 16 March 2018; First published online 23 April 2018

\begin{abstract}
Objective: To examine the relationships between objectively measured sleep patterns (sleep duration, sleep efficiency and bedtime) and sugar-sweetened beverage (SSB) consumption (regular soft drinks, energy drinks, sports drinks and fruit juice) among children from all inhabited continents of the world.

Design: Multinational, cross-sectional study.

Setting: The International Study of Childhood Obesity, Lifestyle and the Environment (ISCOLE).

Subjects: Children ( $n$ 5873) 9-11 years of age.

Results: Sleep duration was 12 min per night shorter in children who reported consuming regular soft drinks 'at least once a day' compared with those who reported consuming 'never' or 'less than once a week'. Children were more likely to sleep the recommended 9-11 h/night if they reported lower regular soft drink consumption or higher sports drinks consumption. Children who reported consuming energy drinks 'once a week or more' reported a 25-min earlier bedtime than those who reported never consuming energy drinks. Children who reported consuming sports drinks ' $2-4 \mathrm{~d}$ a week or more' also reported a 25 -min earlier bedtime compared with those who reported never consuming sports drinks. The associations between sleep efficiency and SSB consumption were not significant. Similar associations between sleep patterns and SSB consumption were observed across all twelve study sites.

Conclusions: Shorter sleep duration was associated with higher intake of regular soft drinks, while earlier bedtimes were associated with lower intake of regular soft drinks and higher intake of energy drinks and sports drinks in this international study of children. Future work is needed to establish causality and to investigate underlying mechanisms.
\end{abstract}

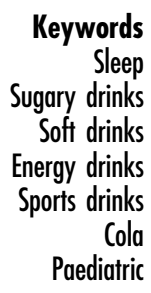

Sugar-sweetened beverages (SSB), defined as any liquids that are sweetened with various forms of added sugars, contribute $10-15 \%$ of children's energy intake and are the primary source of added sugar in their diet ${ }^{(1)}$. SSB consumption is associated with adverse health outcomes including obesity, type 2 diabetes and $\mathrm{CVD}^{(2-4)}$. Putative underlying mechanisms comprise incomplete compensation for liquid energy, adverse glycaemic effects, and increased hepatic metabolism of fructose leading to de novo lipogenesis, production of uric acid and accumulation of visceral and ectopic fat ${ }^{(5)}$. Recent evidence has stimulated public health efforts to reduce SSB consumption as a means of improving childhood weight status and related health outcomes ${ }^{(6)}$. 
Factors associated with SSB consumption in children are numerous $^{(7)}$ and a better understanding of these correlates can inform the development of effective interventions to reduce SSB intake. One factor that has received little attention is the role of sleep, despite accumulating evidence linking insufficient sleep (i.e. short sleep duration and/or poor sleep quality) with obesity and other adverse health outcomes ${ }^{(8,9)}$. The main mechanism linking insufficient sleep to weight gain is through an increase in food intake, especially energy-dense foods ${ }^{(10)}$. Thus, it is plausible that insufficient sleep would be associated with greater intake of SSB in children. Alternatively, SSB consumption may also be associated with insufficient sleep due to the stimulating properties of caffeine that, when consumed near bedtime, may negatively influence sleep.

Studies examining the associations between sleep and SSB consumption are sparse. Prather et al. ${ }^{(11)}$ recently showed that short self-reported sleep duration in adults ( $\leq 5$ and $6 \mathrm{~h} /$ night) was associated with greater intake of sugared caffeinated sodas. Franckle et al ${ }^{(12)}$ reported that children who reported sleeping $<10 \mathrm{~h} / \mathrm{d}$ consumed soda more frequently compared with children who reported sleeping $\geq 10 \mathrm{~h} / \mathrm{d}$. However, to our knowledge, no studies to date have examined whether objectively measured sleep patterns (i.e. sleep duration, sleep efficiency and bedtime) are associated with SSB consumption in children from around the world. Understanding how sleep patterns may be linked to SSB consumption across countries at different levels of economic and human development is important to inform public health policies and tailor interventions that are context- and setting-specific.

The objective of the present study was to investigate the relationships between objectively measured sleep patterns and SSB consumption among a large cross-sectional sample of children from all inhabited continents of the world. We hypothesized that sleep patterns characterized by shorter sleep durations, poorer sleep efficiencies and later bedtimes would be associated with a higher frequency of SSB consumption.

\section{Methods}

\section{Study design and setting}

The International Study of Childhood Obesity, Lifestyle and the Environment (ISCOLE) is a cross-sectional, multinational study designed to examine the relationships between lifestyle behaviours and obesity in twelve study sites located in Australia, Brazil, Canada, China, Colombia, Finland, India, Kenya, Portugal, South Africa, the UK and the USA. These countries represent a wide range of economic development (low to high income), Human Development Index (composite statistic of life expectancy, education and per capita income indicators, used to rank countries into four tiers of human development; 0.509 in Kenya to 0.929 in Australia) and inequality (Gini index of
26.9 in Finland to 63.1 in South Africa) ${ }^{(13)}$. The rationale, design and methods of ISCOLE have previously been published elsewhere ${ }^{(13)}$. The primary sampling frame was schools, which was typically stratified by an indicator of socio-economic status to maximize variability within sites. A standardized protocol was used to collect data across all sites, and all study personnel underwent rigorous training and certification to ensure data quality. Data were collected during the school year at each study site and occurred between September 2011 and December 2013. This study was conducted according to the guidelines laid down in the Declaration of Helsinki. The Pennington Biomedical Research Center Institutional Review Board as well as institutional/ethical review boards at each site approved the study. Written informed consent was obtained from parents/legal guardians and child assent was also obtained.

\section{Participants}

ISCOLE targeted grade levels/classes likely to ensure minimal variability around a mean age of 10 years. All children within the targeted grade level/class in a sampled school were eligible to participate; hence, the sample included 9-11-year-old children. Based on a priori sample size and power calculations ${ }^{(13)}$, each site aimed to recruit a sex-balanced sample of at least 500 children. Of the 7372 children who participated in ISCOLE, a total of 5873 remained in the present analytical data set after excluding participants without valid sleep data ( $n$ 1054), reported level of parental education ( $n$ 273), physical activity data ( $n$ 151), BMI $Z$-score $(n$ 5) and SSB consumption ( $n$ 16). Exclusion of participants for invalid sleep data was mainly due to a wear time of fewer than three nights. Except for significantly higher BMI $Z$-scores, the descriptive characteristics of children who were excluded for missing data did not significantly differ from those who were included in the present analysis.

\section{Measurements}

\section{Sleep patterns}

Sleep duration, sleep efficiency and bedtime were all objectively assessed by $24 \mathrm{~h}$, waist-worn accelerometry. An Actigraph GT3X + accelerometer (ActiGraph LLC, Pensacola, FL, USA) was worn at the waist on an elasticized belt at the right mid-axillary line. Participants were encouraged to wear the accelerometer $24 \mathrm{~h} / \mathrm{d}$ (removing only for water-based activities) for at least seven days, including two weekend days. Data were collected at a sampling rate of $80 \mathrm{~Hz}$, downloaded in $1 \mathrm{~s}$ epochs with the low-frequency extension filter using the ActiLife software version 5.6 or higher, and reintegrated to $60 \mathrm{~s}$ epochs for analysis. Sleep duration (h/night) was estimated using a fully automated algorithm for $24 \mathrm{~h}$ waist-worn accelerometers that was developed and validated for ISCOLE $^{(14,15)}$. This algorithm produces more precise estimates of sleep duration than previous algorithms and 
captures total sleep time from sleep onset to sleep offset, including all epochs and wakefulness after onset ${ }^{(14,15)}$. The weekly sleep duration averages were calculated using only days where valid sleep was accumulated (i.e. total sleep period time $\geq 160 \mathrm{~min} /$ night and $>90 \%$ estimated wear time) and only for participants with at least three nights of valid sleep, including one weekend night (Friday or Saturday). The same device was used to determine sleep efficiency (\%, defined as total sleep episode time divided by sleep period time) and bedtime (hours:minutes, defined as the first five consecutive minutes scored as sleep) $)^{(14-16)}$.

\section{Sugar-sweetened beverage consumption}

Information on SSB consumption was obtained using an FFQ adapted from the Health Behaviour in School-aged Children Survey ${ }^{(13,17)}$. The FFQ asked children to report their consumption of 'Regular cola or soft drinks that contain sugar', 'Energy drinks (Red Bull, Rock Star, Guru, etc.', 'Sports drinks (Gatorade, Powerade, etc.)' and 'Fruit juice', with response options of 'never', 'less than once a week', 'once a week', '2-4 days a week', '5-6 days a week', 'once a day, every day' and 'every day, more than once'. Some categories were combined for analysis due to small sample sizes. A recent study reported a reliability correlation of 0.61 for regular soft drinks, 0.68 for energy drinks, 0.78 for sports drinks and 0.64 for fruit juice among 321 children who repeated this FFQ after an average of 4.9 weeks $^{(18)}$. Given the difficulties in accurately assessing total energy intake in children, we did not measure it in ISCOLE.

\section{Covariates}

Age, sex, highest level of parental education, physical activity level and BMI $Z$-score were included as covariates in statistical models given their association with sleep patterns and/or SSB consumption. Age was computed from birth and observation dates, and sex was recorded on a demographic and family questionnaire. Highest level of parental education was parent-reported and coded into three categories based on the highest level of education attained by either parent: 'did not complete high school', 'completed high school or some college' or 'completed bachelor's or postgraduate degree'. Physical activity data were obtained following a $24 \mathrm{~h}$ protocol using waist-worn accelerometers. After removal of sleep period time from the data file using a published algorithm ${ }^{(14,15)}$, awake nonwear time was defined as at least twenty consecutive minutes of zero activity counts and excluded ${ }^{(19)}$, and moderate-to-vigorous physical activity was defined as all activity $\geq 574$ counts per $15 \mathrm{~s}^{(20)}$. Furthermore, the minimal amount of daytime data that was considered acceptable for inclusion of physical activity data was at least four days with at least $10 \mathrm{~h}$ wear time/d, including at least one weekend day. Based on the average of the monitored days, children were classified as physically active
( $\geq 60 \mathrm{~min} / \mathrm{d}$ on average) or inactive $(<60 \mathrm{~min} / \mathrm{d}$ on average), according to the recommendations of the $\mathrm{WHO}^{(21)}$. Finally, height and body weight were objectively measured using standardized procedures by trained and certified study personnel $^{(13)}$. BMI $\left(\mathrm{kg} / \mathrm{m}^{2}\right)$ was calculated and age- and sex-specific BMI $Z$-scores were computed using reference data from the $\mathrm{WHO}^{(22)}$. Of note, biological maturity was estimated using the maturity offset method; however, because age and weight were included in the maturity offset calculation, biological maturity was not included as a covariate in our analyses.

\section{Statistical analysis}

Statistical analyses were performed using the statistical software packages JMP version 12 and SAS version 9.4. Multilevel mixed-effects models accounting for clustering at the school and study site levels were used to examine the relationships between sleep patterns and SSB consumption. Study sites were considered to have fixed effects, and schools nested within study sites were viewed as having random effects. The denominator degrees of freedom for statistical tests pertaining to fixed effects were calculated using the Kenward and Roger approximation $^{(23)}$. Age, sex, highest level of parental education, meeting WHO physical activity recommendations and BMI $Z$-score were included as covariates in statistical models. Bonferroni corrections were used to account for multiple comparisons. Sleep duration was also dichotomized as $<9 \mathrm{~h} /$ night (sleeping less than recommended) $v$. 9-11 $\mathrm{h} /$ night (meeting the sleep recommendations; reference category), in agreement with current sleep duration guidelines $^{(24,25)}$, to calculate the odds of meeting the sleep duration recommendations for each of the four SSB consumption variables (treated as categorical variables). A total of thirty-seven children slept more than $11 \mathrm{~h} /$ night (long sleepers) and were thus excluded from this analysis. However, keeping them or excluding them did not impact the results found. We also examined associations between sleep patterns and SSB consumption according to countrylevel World Bank classification of economic development ${ }^{(26)}$. $P$ values of less than 0.05 were considered statistically significant.

\section{Results}

Table 1 presents descriptive characteristics of the sample. The mean sleep duration was $8.8 \mathrm{~h} /$ night (with Portugal having the shortest sleep duration of the countries examined ( $8.3 \mathrm{~h}$ ) and the UK the longest (9.5 h)) and $58 \%$ of children slept less than the recommended 9-11 h/night. Children were very sleep efficient $(96 \cdot 2 \%$ sleep efficiency on average) and had a mean bedtime of 22:18 (latest mean bedtime in Portugal (23:15) and earliest in Kenya (21:41)). A total of $11.6 \%$ of children reported that they consumed regular cola or soft drinks once per day or more (ranging 
Table 1 Descriptive characteristics of participants: children aged 9-11 years ( $n$ 5873) from twelve study sites around the world, International Study of Childhood Obesity, Lifestyle and the Environment (ISCOLE), September 2011-December 2013

\begin{tabular}{|c|c|}
\hline \multicolumn{2}{|l|}{ Age (years) } \\
\hline Mean & $10 \cdot 4$ \\
\hline SD & 0.6 \\
\hline \multicolumn{2}{|l|}{$\operatorname{Sex}(\%)$} \\
\hline Boys & $45 \cdot 1$ \\
\hline Girls & 54.9 \\
\hline \multicolumn{2}{|l|}{$\mathrm{BMI}\left(\mathrm{kg} / \mathrm{m}^{2}\right)$} \\
\hline Mean & $18 \cdot 4$ \\
\hline SD & 3.4 \\
\hline Obesity (\%)† & $12 \cdot 2$ \\
\hline \multicolumn{2}{|l|}{ Highest parental education (\%) } \\
\hline Did not complete high school & $19 \cdot 7$ \\
\hline Completed high school or some college & 42.5 \\
\hline Completed bachelor's or postgraduate degree & $37 \cdot 8$ \\
\hline Meeting physical activity guidelines (\%) & $45 \cdot 8$ \\
\hline \multicolumn{2}{|l|}{ Sleep duration (h/night) } \\
\hline Mean & $8 \cdot 8$ \\
\hline \multirow{2}{*}{\multicolumn{2}{|c|}{ Sleep efficiency (\%) }} \\
\hline & \\
\hline Mean & $96 \cdot 2$ \\
\hline $\mathrm{SD}$ & $1 . \overline{4}$ \\
\hline Bedtime (hours:minutes) & $22: 18$ \\
\hline \multicolumn{2}{|l|}{ Consumption of regular cola or soft drinks (\%) } \\
\hline Never & $15 \cdot 1$ \\
\hline$<$ Once/week & $27 \cdot 2$ \\
\hline Once/week & 23.4 \\
\hline $2-4 \mathrm{~d} /$ week & $16 \cdot 5$ \\
\hline $5-6 \mathrm{~d} /$ week & 6.2 \\
\hline$\geq$ Once/d & 11.6 \\
\hline \multicolumn{2}{|l|}{ Consumption of energy drinks (\%) } \\
\hline Never & $75 \cdot 9$ \\
\hline$<$ Once/week & $9 \cdot 6$ \\
\hline$\geq$ Once/week & 14.5 \\
\hline \multicolumn{2}{|l|}{ Consumption of sports drinks (\%) } \\
\hline Never & $45 \cdot 1$ \\
\hline$<$ Once/week & 21.0 \\
\hline Once/week & $12 \cdot 0$ \\
\hline $2-4 \mathrm{~d} /$ week or more & 21.9 \\
\hline \multicolumn{2}{|l|}{ Consumption of fruit juice (\%) } \\
\hline Never or < once/week & $19 \cdot 7$ \\
\hline Once/week & $14 \cdot 0$ \\
\hline $2-4 \mathrm{~d} /$ week & $16 \cdot 7$ \\
\hline $5-6 \mathrm{~d} /$ week & 11.9 \\
\hline Once/d, every day & $15 \cdot 3$ \\
\hline Every day, >once & 22.4 \\
\hline
\end{tabular}

†Obesity defined according to the WHO criteria ${ }^{(22)}$.

from $1.1 \%$ reporting consuming regular soda or soft drinks once per day or more in Finland to $31.6 \%$ in South Africa). Approximately three-quarters of children reported never consuming energy drinks (ranging from 46\% reporting 'never' consuming energy drinks in South Africa to 95\% in Finland). Approximately $45 \%$ of the sample reported that they never consumed sports drinks (ranging from 9\% reporting 'never' consuming sports drinks in the USA to $80 \%$ in Finland). Finally, $22.4 \%$ of children reported drinking fruit juice more than once per day (ranging from $6 \%$ reporting drinking fruit juice more than once per day in China to $47 \%$ in Colombia).

We did not find significant sex interactions in the associations between sleep patterns and SSB consumption across study sites; therefore, results were pooled for presentation. Figures 1-3 show sleep patterns across levels of consumption of SSB in this sample of children. There was a significant negative trend in sleep duration across levels of consumption of regular soft drinks (Fig. 1(a)). Sleep duration was $12 \mathrm{~min}$ shorter in children who reported consuming regular soft drinks ' $\geq$ once/d' compared with those who reported consuming regular soft drinks 'never' or '< once/week'. We also observed significant positive trends between bedtime and consumption of regular soft drinks (Fig. 3(a)), and significant negative trends between bedtime and consumption of energy drinks (Fig. 3(b)) and sports drinks (Fig. 3(c)). In particular, we observed a 25 -min earlier bedtime in children who reported consuming energy drinks ' $\geq$ once/ week' compared with those who reported 'never' consuming energy drinks. Likewise, we found a 25-min earlier bedtime in children who reported consuming sports drinks '2 $-4 \mathrm{~d}$ /week or more' compared with those reporting 'never'. The other associations between sleep patterns and SSB consumption were not significant. We also did not find a significant World Bank classification of economic development-by-sleep pattern interaction for SSB consumption, suggesting that the associations did not differ between sites (e.g. low- $v$. high-income countries).

Table 2 presents the odds of meeting the sleep duration recommendation of $9-11 \mathrm{~h} /$ night (reference category) for each of the four SSB consumption variables. The cut-points for the SSB consumption variables were chosen based on the distribution of the data, to maximize power. Children who reported drinking regular soft drinks 'once/ week or more' were less likely to sleep the recommended amount $(\mathrm{OR}=0.79$, 95\% CI 0.71, 0.88) compared with those who reported consuming regular soft drinks 'never or < once/week'. In contrast, higher odds of meeting the sleep duration recommendation were observed in children reporting consuming sports drinks '< once/week or more' $(\mathrm{OR}=1 \cdot 26,95 \%$ CI 1.13, 1.39) compared with those reporting never consuming sports drinks. Finally, children reporting drinking fruit juice 'once/week or more' had higher odds of meeting the sleep duration recommendation $(\mathrm{OR}=1 \cdot 23,95 \% \mathrm{CI} 1.08,1.40)$ than those indicating drinking fruit juice 'never or < once/week'.

\section{Discussion}

To our knowledge, the present study is the first to examine the relationships between sleep patterns and SSB consumption in children from twelve countries varying widely in levels of economic and human development. Collectively, we observed shorter sleep durations with higher consumption of regular soft drinks. Children were also more likely to sleep the recommended 9-11 h/night if they reported lower regular soft drink consumption or higher sports drinks or fruit juice consumption. We also observed that later bedtimes were associated with higher 
(a)

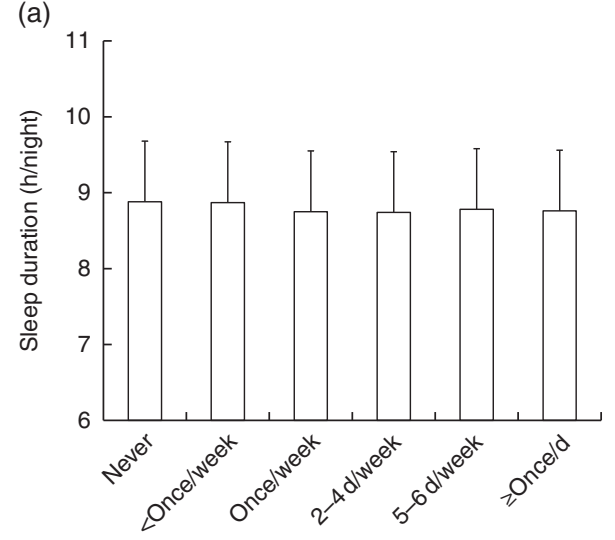

(c)

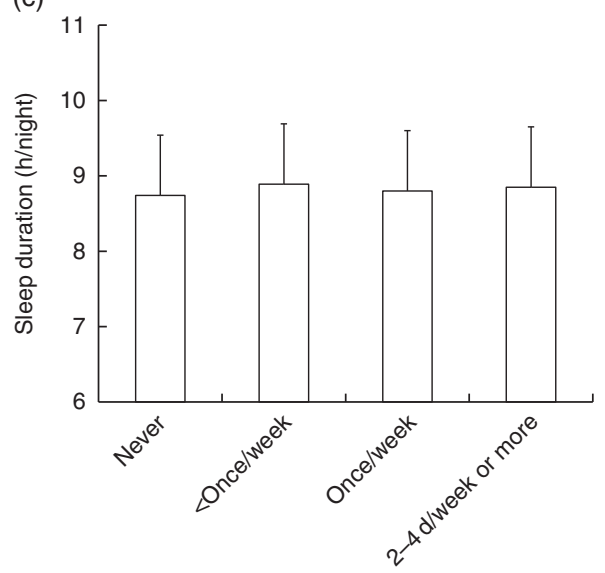

(b)

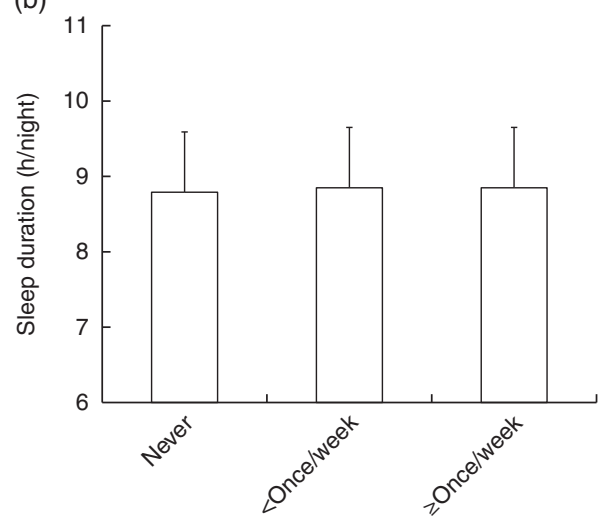

(d)

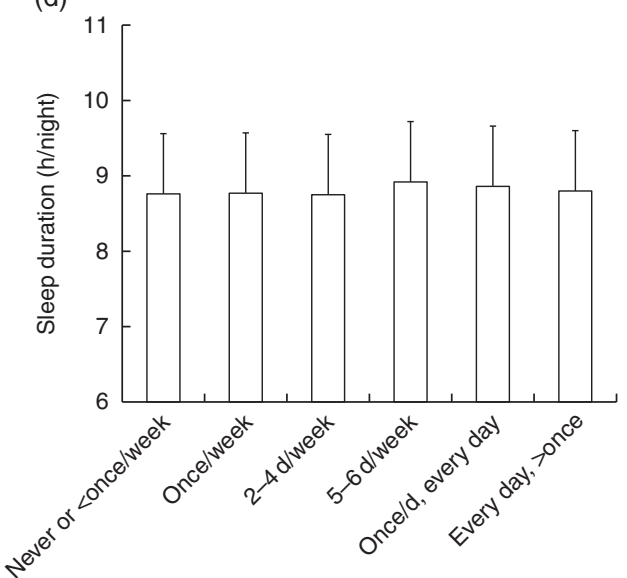

Fig. 1 Sleep duration across levels of consumption of (a) regular cola or soft drinks $(P$ for linear trend $<0.01)$, (b) energy drinks ( $P$ for linear trend NS), (c) sports drinks ( $P$ for linear trend NS) and (d) fruit juice $(P$ for linear trend NS) among children aged 9-11 years $(n 5873)$ from twelve study sites around the world, International Study of Childhood Obesity, Lifestyle and the Environment (ISCOLE), September 2011-December 2013. Data are presented as mean values with standard deviations represented by vertical bars. Age, sex, highest level of parental education, meeting physical activity guidelines and BMI Z-score were included as covariates

consumption of regular soft drinks. Conversely, later bedtimes were also associated with lower consumption of energy drinks and sports drinks. There was no association between sleep efficiency and SSB consumption. Similar associations between sleep patterns and SSB consumption were observed across all twelve study sites.

The present findings are in line with previous studies that have reported a significant relationship between sleep duration and SSB consumption. For example, short selfreported sleep duration ( $\leq 5$ and $6 \mathrm{~h} /$ night) has been shown to be associated with higher intake of sugared caffeinated sodas among adults in the USA ${ }^{(11)}$. In children, Franckle et al. $^{(12)}$ reported that students in two Massachusetts communities who reported sleeping $<10 \mathrm{~h} / \mathrm{d}$ consumed soda more frequently compared with students who reported $\geq 10 \mathrm{~h}$ sleep/d. However, no significant association was reported with fruit juice in their study, in agreement with the present work. Similarly, Pérez-Farinós et al. ${ }^{(27)}$ reported that short sleep duration $(<9.9 \mathrm{~h} / \mathrm{d})$ was associated with a greater frequency of consumption of soft drinks containing sugar but not with fruit juice in Spanish children. No studies have looked at the association of sleep quality or sleep timing (e.g. bedtime or chronotype) with SSB consumption in children.

Among the three sleep characteristics examined in the present study, bedtime was most strongly associated with SSB consumption. Similar to short sleep duration, later bedtimes were associated with greater consumption of regular soft drinks. Yet, earlier bedtimes were also associated with greater consumption of energy drinks and sports drinks. Although this may seem counter-intuitive, a greater frequency of consumption of energy and sports drinks may be a proxy for a healthier lifestyle in general. For instance, it is possible that active children go to bed earlier and may consume energy and sports drinks more frequently during the day for their physical activities (or other reasons). Energy and sports drinks may be seen as 'good' by children and parents despite the fact they are not healthy options according to public health authorities. Sampasa-Kanyinga and Chaput have recently reported that female adolescents who meet the recommendation of $\geq 60$ min of moderate-to-vigorous physical activity daily are more likely to report consuming energy drinks than 
(a)

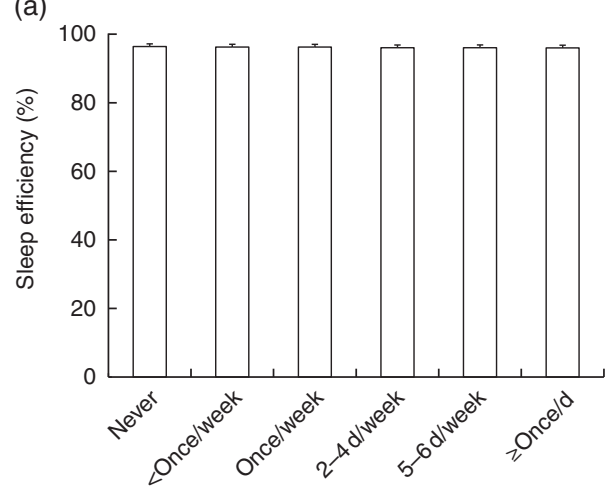

(c)

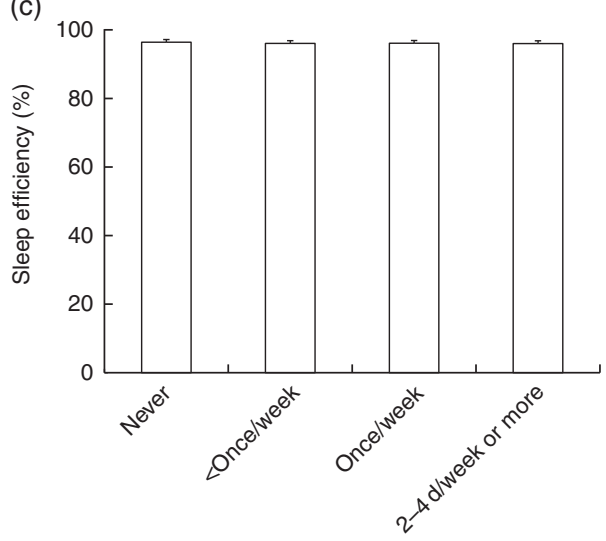

(b)

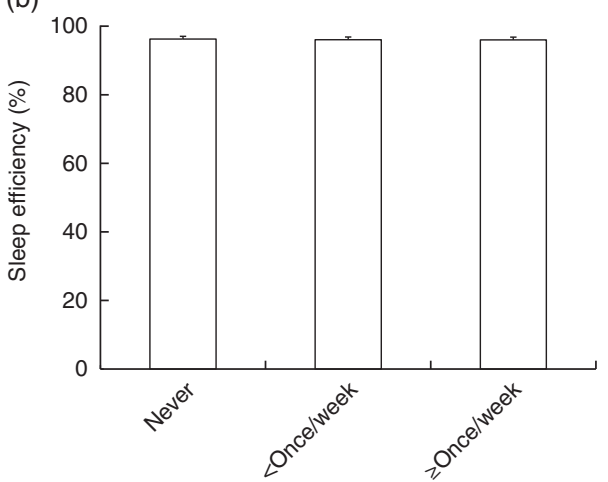

(d)

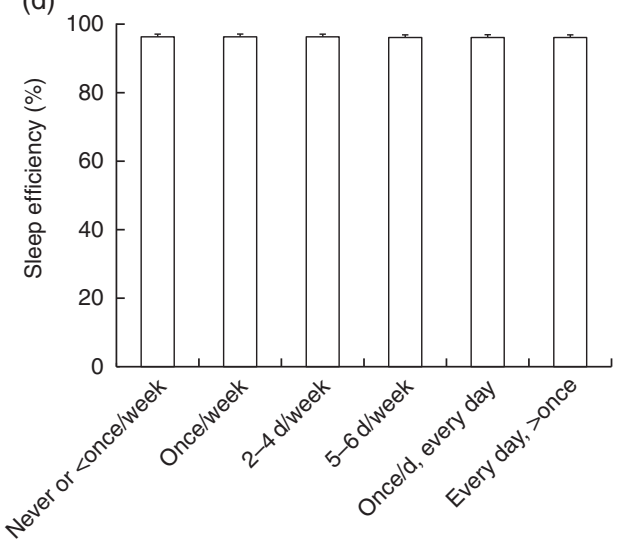

Fig. 2 Sleep efficiency across levels of consumption of (a) regular cola or soft drinks ( $P$ for linear trend NS), (b) energy drinks ( $P$ for linear trend NS), (c) sports drinks ( $P$ for linear trend NS) and (d) fruit juice $(P$ for linear trend NS) among children aged 9-11 years $(n 5873)$ from twelve study sites around the world, International Study of Childhood Obesity, Lifestyle and the Environment (ISCOLE), September 2011-December 2013. Data are presented as mean values with standard deviations represented by vertical bars. Age, sex, highest level of parental education, meeting physical activity guidelines and BMI Z-score were included as covariates

those who do not meet this recommendation ${ }^{(28)}$. Conversely, late bedtimes are generally associated with more screen time and energy-dense food snacking ${ }^{(29,30)}$. The present data suggest that a greater consumption of sugarsweetened soft drinks is linked to later bedtimes in children from around the world.

Reverse causation is always a possibility with crosssectional study designs. Thus, it is possible that SSB consumption may also impact sleep patterns, especially due to the stimulating properties of caffeine that can disrupt sleep. Although caffeine use is well known to reduce sleep quality (especially when consumed in the hours before bedtime), we did not find a significant association between sleep efficiency and SSB consumption in the present study. One explanation is the ceiling effect observed for sleep efficiency in this sample of children (mean value of $96 \%$ ). It is indeed difficult to find significant associations with small inter-individual variations in the data. Although children have high sleep efficiency values in general (e.g. compared with adults), the waist-worn accelerometer protocol used in ISCOLE tends to also overestimate sleep efficiency compared with wrist-worn devices ${ }^{(31)}$. Future studies using more sensitive measures of sleep quality are thus required to confirm our findings. Longitudinal studies will also be needed to determine the directionality in the findings, including information about when children routinely consume the different SSB (e.g. during the day or near bedtime).

The current study included sites from countries varying widely in levels of economic and human development. However, we did not find a significant World Bank classification of economic development-by-sleep pattern interaction for SSB consumption, suggesting that the associations were similar across study sites. Although limited, the current evidence on sleep patterns as it relates to SSB consumption is mainly from high-income countries. It is thus reassuring to observe herein the same associations all over the world, thereby making future intervention strategies more generalizable. However, ISCOLE did not contain nationally representative data, so it would be prudent to design interventions that are context- and setting-specific to optimize success.

Determinants of SSB consumption in children are numerous and include things such as a child's preference for SSB, screen time and snack consumption, lower 
(a)

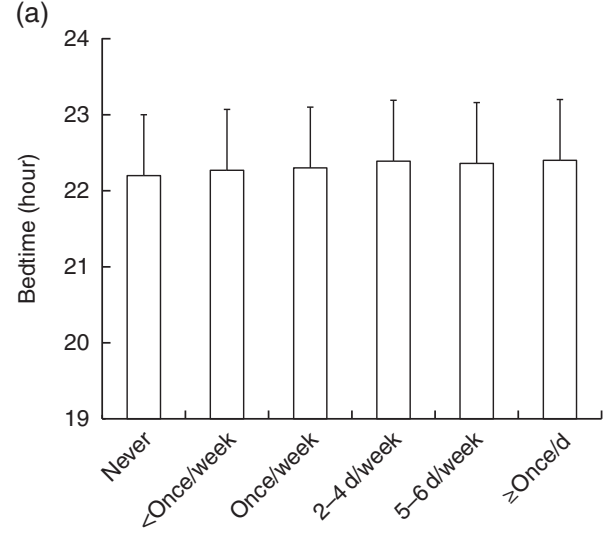

(c)

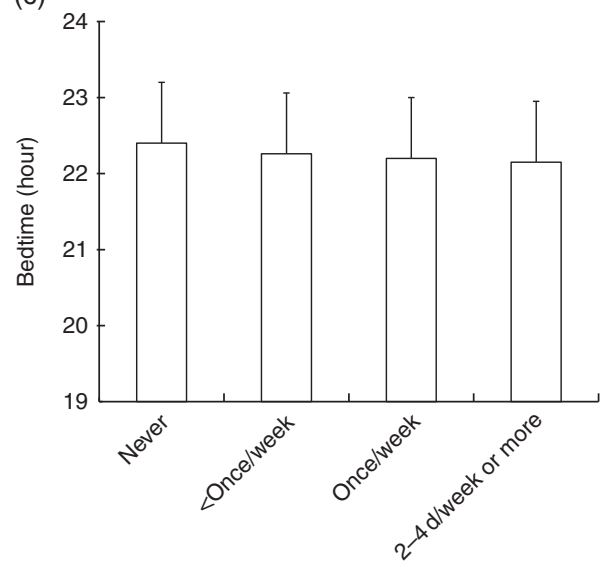

(b)

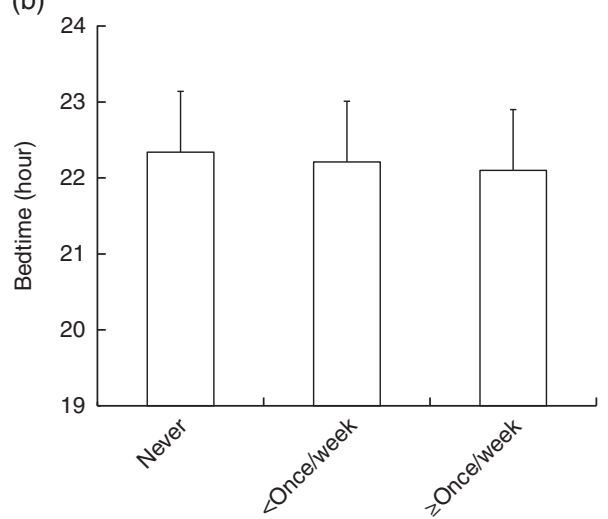

(d)

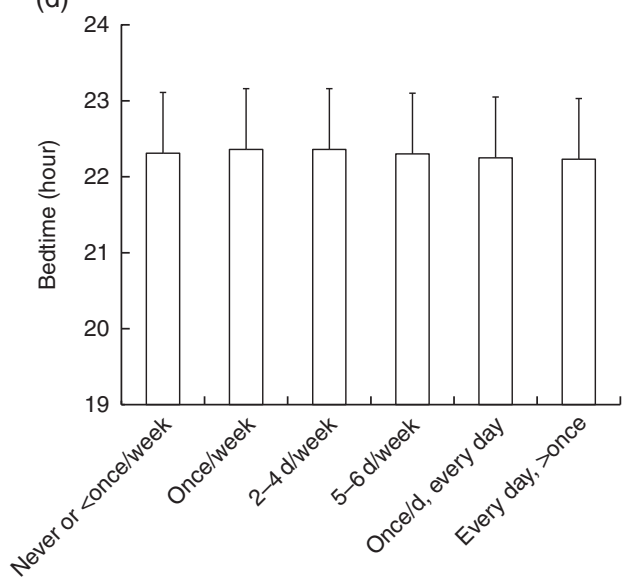

Fig. 3 Bedtime across levels of consumption of (a) regular cola or soft drinks $(P$ for linear trend $<0.01)$, (b) energy drinks $(P$ for linear trend $<0.01)$, (c) sports drinks $(P$ for linear trend $<0.01)$ and $(d)$ fruit juice $(P$ for linear trend NS) among children aged 9-11 years $(n$ 5873) from twelve study sites around the world, International Study of Childhood Obesity, Lifestyle and the Environment (ISCOLE), September 2011-December 2013. Data are presented as mean values with standard deviations represented by vertical bars. Age, sex, highest level of parental education, meeting physical activity guidelines and BMI Z-score were included as covariates

Table 2 Odds for meeting the sleep duration recommendation of $9-11 \mathrm{~h} /$ night (compared with $<9 \mathrm{~h} /$ night), for each of the sugarsweetened beverage (SSB) consumption variables, among children aged $9-11$ years $(n 5873)$ from twelve study sites around the world, International Study of Childhood Obesity, Lifestyle and the Environment (ISCOLE), September 2011-December 2013

\begin{tabular}{lcc}
\hline SSB variable & OR & $95 \% \mathrm{Cl}$ \\
\hline Regular cola or soft drinks & & \\
$\quad$ Never or <once/week & 1.00 & Ref. \\
$\quad$ Once/week or more & 0.79 & $0.71,0.88^{*}$ \\
$\begin{array}{l}\text { Energy drinks } \\
\quad \text { Never }\end{array}$ & 1.00 & Ref. \\
$\quad \begin{array}{l}<\text { Once/week or more } \\
\text { Sports drinks }\end{array}$ & 1.08 & $0.96,1.21$ \\
$\quad$ Never \\
$\quad<$ Once/week or more & 1.00 & Ref. \\
Fruit juice & 1.26 & $1.13,1.39^{*}$ \\
$\quad$ Never or <once/week & 1.00 & Ref. \\
$\quad$ Once/week or more & 1.23 & $1.08,1.40^{*}$ \\
\hline
\end{tabular}

Ref., reference category.

Age, sex, highest level of parental education, meeting physical activity guidelines and BMI $Z$-score were included as covariates in statistical models. ${ }^{\star} P<0.05$.

parental socio-economic status, parental role modelling, using food as a reward or living near a fast-food/convenience store $^{(7)}$. Likewise, reasons for having inadequate sleep patterns are diverse and can include a lack of parental monitoring or rules about bedtime in the household, artificial light exposure before bedtime, electronic devices in the bedroom, unfavourable sleep environment, cultural factors, etc. Sleep duration of school-aged children is also largely influenced by the start of the school day and bedtime is therefore a key determinant of total sleep duration in such a context. A better understanding of the determinants of SSB consumption and sleep patterns is important to inform the development of effective interventions aimed at reducing SSB consumption and improving sleep hygiene of children.

The present study has several strengths and limitations that warrant discussion. An important strength is the large multinational sample of children from low- to high-income countries across several regions of the world. We also used a highly standardized measurement protocol, objective sleep measurements and a rigorous quality control programme to ensure high-quality data across all sites ${ }^{(13)}$. However, our results need to be interpreted in the light of the following limitations. First, the cross-sectional nature of the data precludes inferences about causality or 
temporality. Second, accelerometers may be limited in their ability to properly distinguish between sleep and wake states, as they are based on movement detection, and waistworn accelerometers have been shown to overestimate absolute sleep duration and sleep efficiency compared with wrist-worn devices ${ }^{(31)}$. Third, ISCOLE was not designed to provide nationally representative data and therefore the degree to which the results are generalizable to the studied countries is not known. Fourth, the narrow age range limits our ability to infer our findings to other age groups and it is possible that different patterns would be observed in adolescents or adults for example. Fifth, only the frequency of SSB consumption was reported and information on energy intake (kilojoules) was not captured in ISCOLE. Reliability correlation coefficients of 0.61 (regular soft drinks), 0.68 (energy drinks), 0.78 (sports drinks) and 0.64 (fruit juice) have been reported in children who repeated this FFQ after an average of 4.9 weeks. Also, the FFQ used did not distinguish between fruit juice with or without added sugar. Finally, the potential confounding effects of unmeasured variables cannot be discounted.

\section{Conclusion}

In conclusion, findings from the current study show that shorter sleep duration was associated with higher intake of regular soft drinks, while earlier bedtimes were associated with lower intake of regular soft drinks and higher intake of energy drinks and sports drinks in this large multinational study of children. Further studies using longitudinal research designs are needed to better understand the prospective associations among sleep patterns and SSB consumption in children.

\section{Acknowledgements}

Acknowledgements: The authors wish to thank the ISCOLE External Advisory Board and the ISCOLE participants and their families who made this study possible. The ISCOLE Research Group includes the following. Coordinating Center, Pennington Biomedical Research Center: Peter T. Katzmarzyk, PhD (Co-Principal Investigator (PI)); Timothy S. Church, MD, PhD (Co-PI); Denise G. Lambert, RN (Project Manager); Tiago Barreira, PhD; Stephanie Broyles, PhD; Ben Butitta, BS; Catherine Champagne, PhD, RD; Shannon Cocreham, MBA; Kara D. Denstel, MPH; Katy Drazba, MPH; Deirdre Harrington, $\mathrm{PhD}$; William Johnson, PhD; Dione Milauskas, MS; Emily Mire, MS; Allison Tohme, MPH; Ruben Rodarte MS, MBA. Data Management Center, Wake Forest University: Bobby Amoroso, BS; John Luopa, BS; Rebecca Neiberg, MS; Scott Rushing, BS. Australia, University of South Australia: Timothy Olds, PhD (Site Co-PI); Carol Maher, PhD (Site Co-PI); Lucy Lewis, PhD; Katia Ferrar, BPhysio (Hons); Effie Georgiadis, BPsych; Rebecca Stanley, BAppSc (OT) Hons. Brazil, Centro de Estudos do Laboratório de Aptidão Física de São Caetano do Sul (CELAFISCS): Victor Keihan Rodrigues Matsudo, MD, $\mathrm{PhD}$ (Site PI); Sandra Matsudo, MD, PhD; Timoteo Araujo, MSc; Luis Carlos de Oliveira, MSc; Luis Fabiano, BSc; Diogo Bezerra, BSc; Gerson Ferrari, MSc. Canada, Children's Hospital of Eastern Ontario Research Institute: Mark S. Tremblay, $\mathrm{PhD}$ (Site Co-PI); Jean-Philippe Chaput, $\mathrm{PhD}$ (Site Co-PI); Priscilla Bélanger, MA; Mike Borghese, MSc; Charles Boyer, MA; Allana LeBlanc, PhD; Claire Francis, MSc; Geneviève Leduc, PhD. China, Tianjin Women's and Children's Health Center: Pei Zhao, MD (Site Co-PI); Gang Hu, MD, PhD (Site Co-PI); Chengming Diao, MD; Wei Li, MD; Weiqin Li, MSc; Enqing Liu, MD; Gongshu Liu, MD; Hongyan Liu, MD; Jian Ma, MD; Yijuan Qiao, MD; Huiguang Tian, PhD; Yue Wang, MD; Tao Zhang, MSc; Fuxia Zhang, MD. Colombia, Universidad de los Andes: Olga Sarmiento, MD, PhD (Site PI); Julio Acosta; Yalta Alvira, BS; Maria Paula Diaz; Rocio Gamez, BS; Maria Paula Garcia; Luis Guillermo Gómez; Lisseth Gonzalez; Silvia Gonzalez, RD; Carlos Grijalba, MD; Leidys Gutierrez; David Leal; Nicolas Lemus; Etelvina Mahecha, BS; Maria Paula Mahecha; Rosalba Mahecha, BS; Andrea Ramirez, MD; Paola Rios, MD; Andres Suarez; Camilo Triana. Finland, University of Helsinki: Mikael Fogelholm, ScD (Site-PI); Elli Hovi, BS; Jemina Kivelä; Sari Räsänen, BS; Sanna Roito, BS; Taru Saloheimo, MS; Leena Valta. India, St. Johns Research Institute: Anura Kurpad, MD, $\mathrm{PhD}$ (Site Co-PI); Rebecca Kuriyan, PhD (Site Co-PI); Deepa P. Lokesh, BSc; Michelle Stephanie D'Almeida, BSc; Annie Mattilda, MSc; Lygia Correa, BSc; Vijay Dakshina Murthy, BSc. Kenya, Kenyatta University: Vincent Onywera, $\mathrm{PhD}$ (Site Co-PI); Mark S. Tremblay, PhD (Site Co-PI); Lucy-Joy Wachira, PhD; Stella Muthuri, PhD. Portugal, University of Porto: Jose Maia, PhD (Site PI); Alessandra da Silva Borges, BA; Sofia Oliveira Sá Cachada, MSc; Raquel Nichele de Chaves, MSc; Thayse Natacha Queiroz Ferreira Gomes, PhD, MSc; Sara Isabel Sampaio Pereira, BA; Daniel Monteiro de Vilhena e Santos, PhD; Fernanda Karina dos Santos, MSc; Pedro Gil Rodrigues da Silva, BA; Michele Caroline de Souza, MSc. South Africa, University of Cape Town: Vicki Lambert, $\mathrm{PhD}$ (Site PI); Matthew April, BSc (Hons); Monika Uys, BSc (Hons); Nirmala Naidoo, MSc; Nandi Synyanya; Madelaine Carstens; BSc (Hons). UK, University of Bath: Martyn Standage, $\mathrm{PhD}$ (Site PI); Sean Cumming, PhD; Clemens Drenowatz, PhD; Lydia Emm, MSc; Fiona Gillison, PhD; Julia Zakrzewski, PhD. USA, Pennington Biomedical Research Center: Catrine Tudor-Locke, PhD (Site-PI); Ashley Braud; Sheletta Donatto, MS, LDN, RD; Corbin Lemon, BS; Ana Jackson, BA; Ashunti Pearson, MS; Gina Pennington, BS, LDN, RD; Daniel Ragus, BS; Ryan Roubion; John Schuna Jr, PhD; Derek Wiltz. The ISCOLE External Advisory Board: Alan Batterham, PhD, Teesside University; Jacqueline Kerr, PhD, University of California, San Diego; Michael Pratt, MD, Centers for Disease Control and Prevention; Angelo Pietrobelli, MD, Verona University Medical School. Financial support: ISCOLE was funded by The Coca-Cola Company. With the exception of requiring that the study be global in nature, the funder had 
no role in the design and conduct of the study; collection, management, analysis and interpretation of the data; preparation, review or approval of the manuscript; and decision to submit the manuscript for publication. Conflict of interest: None. Authorship: J.-P.C. and H.S.-K. conceived the paper. J.-P.C. performed the statistical analyses. J.-P.C. wrote the first draft of the article. All authors critically reviewed the manuscript, provided feedback and approved the final submission. Ethics of human subject participation: This study was conducted according to the guidelines laid down in the Declaration of Helsinki. The Pennington Biomedical Research Center Institutional Review Board as well as institutional/ ethical review boards at each site approved the study. Written informed consent was obtained from parents/legal guardians and child assent was also obtained.

\section{References}

1. Hu FB \& Malik VS (2010) Sugar-sweetened beverages and risk of obesity and type 2 diabetes: epidemiologic evidence. Physiol Behav 100, 47-54.

2. Scharf RJ \& DeBoer MD (2016) Sugar-sweetened beverages and children's health. Annu Rev Public Health 37, 273-293.

3. Bes-Rastrollo M, Sayon-Orea C, Ruiz-Canela M et al. (2016) Impact of sugars and sugar taxation on body weight control: a comprehensive literature review. Obesity (Silver Spring) 24, 1410-1426.

4. Keller A \& Bucher Della Torre S (2015) Sugar-sweetened beverages and obesity among children and adolescents: a review of systematic literature reviews. Child Obes $\mathbf{1 1}$, 338-346.

5. Malik VS \& Hu FB (2015) Fructose and cardiometabolic health: what the evidence from sugar-sweetened beverages tells us. J Am Coll Cardiol 66, 1615-1624.

6. Le Bodo Y, Paquette MC, Vallières M et al. (2015) Is sugar the new tobacco? Insights from laboratory studies, consumer surveys and public health. Curr Obes Rep 4, 111-121.

7. Mazarello Paes V, Hesketh K, O'Malley C et al. (2015) Determinants of sugar-sweetened beverage consumption in young children: a systematic review. Obes Rev 16, 903-913.

8. Chaput JP, Gray CE, Poitras VJ et al. (2016) Systematic review of the relationships between sleep duration and health indicators in school-aged children and youth. Appl Physiol Nutr Metab 41, 6 Suppl. 3, S266-S282.

9. Chaput JP (2016) Is sleep deprivation a contributor to obesity in children? Eat Weight Disord 21, 5-11.

10. Chaput JP (2014) Sleep patterns, diet quality and energy balance. Physiol Behav 134, 86-91.

11. Prather AA, Leung CW, Adler NE et al. (2016) Short and sweet: associations between self-reported sleep duration and sugar-sweetened beverage consumption among adults in the United States. Sleep Health 2, 272-276.

12. Franckle RL, Falbe J, Gortmaker S et al. (2015) Insufficient sleep among elementary and middle school students is linked with elevated soda consumption and other unhealthy dietary behaviors. Prev Med 74, 36-41.

13. Katzmarzyk PT, Barreira TV, Broyles ST et al. (2013) The International Study of Childhood Obesity, Lifestyle and the Environment (ISCOLE): design and methods. BMC Public Health 13, 900.
14. Tudor-Locke C, Barreira TV, Schuna JM Jr et al. (2014) Fully automated waist-worn accelerometer algorithm for detecting children's sleep-period time separate from 24-h physical activity or sedentary behaviors. Appl Physiol Nutr Metab 39, $53-57$.

15. Barreira TV, Schuna JM Jr, Mire EF et al. (2015) Identifying children's nocturnal sleep using a 24-h waist accelerometry. Med Sci Sports Exerc 47, 937-943.

16. Tudor-Locke C, Mire EF, Barreira TV et al. (2015) Nocturnal sleep-related variables from 24-h free-living waist-worn accelerometry: International Study of Childhood Obesity, Lifestyle and the Environment. Int J Obes Suppl 5, Suppl. 2, S47-S52.

17. Currie C, Gabhainn SN, Godeau E et al. (2008) Inequalities in Children's Health: HBSC International Report from the 2005/2006 Survey. Health Policy for Children and Adolescents no. 5. Copenhagen: WHO Regional Office for Europe.

18. Saloheimo T, Gonzalez SA, Erkkola M et al. (2015) The reliability and validity of a short food frequency questionnaire among 9-11-year old: a multinational study on three middle-income and high-income countries. Int J Obes Suppl 5, Suppl. 2, S22-S28.

19. Mark AE \& Janssen I (2008) Dose-response relation between physical activity and blood pressure in youth. Med Sci Sports Exerc 40, 1007-1012.

20. Evenson KR, Catellier DJ, Gill K et al. (2008) Calibration of two objective measures of physical activity for children. J Sports Sci 26, 1557-1565.

21. World Health Organization (2010) Global Recommendations on Physical Activity for Health, p. 58. Geneva: WHO.

22. de Onis M, Onyango AW, Borghi E et al. (2007) Development of a WHO growth reference for school-aged children and adolescents. Bull World Health Organ 85, 660-667.

23. Kenward MG \& Roger JH (1997) Small sample inference for fixed effects from restricted maximum likelihood. Biometrics 53, 983-997.

24. Hirshkowitz M, Whiton K, Albert SM et al. (2015) National Sleep Foundation's updated sleep duration recommendations: final report. Sleep Health 1, 233-243.

25. Tremblay MS, Carson V, Chaput JP et al. (2016) Canadian 24-hour movement guidelines for children and youth: an integration of physical activity, sedentary behaviour, and sleep. Appl Physiol Nutr Metab 41, 6 Suppl. 3, S311-S327.

26. World Bank (2012) World Development Indicators. Washington, DC: The World Bank

27. Pérez-Farinós N, Villar-Villalba C, López Sobaler AM et al. (2017) The relationship between hours of sleep, screen time and frequency of food and drink consumption in Spain in the 2011 and 2013 ALADINO: a cross-sectional study. BMC Public Health 17, 33.

28. Sampasa-Kanyinga H \& Chaput JP (2017) Consumption of sugar-sweetened beverages and energy drinks and adherence to physical activity and screen time recommendations among adolescents. Int J Adolesc Med Health 29, 20150098.

29. Adamo KB, Wilson S, Belanger K et al. (2013) Later bedtime is associated with greater daily energy intake and screen time in obese adolescents independent of sleep duration. J Sleep Disord Ther 2, 126.

30. Thivel D, Tremblay MS \& Chaput JP (2013) Modern sedentary behaviors favor energy consumption in children and adolescents. Curr Obes Rep 2, 50-57.

31. Hjorth MF, Chaput JP, Damsgaard CT et al. (2012) Measure of sleep and physical activity by a single accelerometer: can a waist-worn Actigraph adequately measure sleep in children? Sleep Biol Rhythms 10, 328-335. 